\title{
Cold mirror based on High-Low-High refractive index dielectric materials
}

\author{
V.V. Elyutin ${ }^{1}$, M.A. Butt ${ }^{1}$, S.N. Khonina ${ }^{1,2}$ \\ ${ }^{1}$ Samara National Research University, 34 Moskovskoe Shosse, 443086, Samara, Russia \\ ${ }^{2}$ Image Processing Systems Institute - Branch of the Federal Scientific Research Centre "Crystallography and Photonics" of Russian Academy of Sciences, 151 \\ Molodogvardeyskaya st., 443001, Samara, Russia
}

\begin{abstract}
In this paper, a design for a multilayer dielectric cold mirror based on $\mathrm{TiO}_{2} / \mathrm{SiO}_{2}$ and $\mathrm{TiO}_{2} / \mathrm{MgF}_{2}$ alternating layers is presented. A cold mirror is a specific dielectric mirror that reflects the complete visible light spectrum whereas transmitting the infrared wavelengths. These mirrors are designed for an incident angle of $45^{\circ}$, and are modeled with multilayer dielectric coatings similar to interference filters. Our designed mirror based on $\mathrm{TiO}_{2} / \mathrm{SiO}_{2}$ shows an average transmission of less than $5 \%$ in the spectrum range of $425-610 \mathrm{~nm}$ whereas it has an average transmission of $95 \%$ in the spectrum range of $710-1500 \mathrm{~nm}$.
\end{abstract}

Keywords: Cold mirror; $\mathrm{TiO}_{2} ; \mathrm{MgF}_{2} ; \mathrm{SiO}_{2}$; dielectric materials

\section{Introduction}

Thin film optics is a well-developed technology and many devices such as passband filters, stopband filters, polarizers and reflectors are successfully developed with the help of multilayer dielectric thin films [1-4]. These optical elements comprise of alternating layers of high and low refractive index materials with specific thicknesses and awareness of their refractive index and absorption. Multilayer dielectric filters are based on the principle of multiple reflections that takes place between the interfaces of high and low index materials. Distributed Bragg Reflectors (DBRs) are one of the widely used filters which are quarter wave thick of the center wavelength. The high reflection region of a DBR is known as the DBR stopband and can be obtained by the refractive index contrast between the constituent layers [5]. A cold mirror is a specific dielectric mirror that reflects the visible light spectrum while transmits the infrared wavelengths. These mirrors work on the principle of multiple reflections between high and low index material interface. The visible spectrum of light spans $\sim 380-770 \mathrm{~nm}$ and the region beyond $770 \mathrm{~nm}$ in the near infrared, which is heat. Radiations from a tungsten lamp contain at least six times as much heat as useful light in the visible spectrum. The term cold light defines the radiation in which the IR spectrum is removed [6].

A hot mirror is just the opposite of cold mirror which is designed to reflect infrared region while transmits the visible portion of the beam. These mirrors can separate visible light from UV and NIR which helps in separating the heat from the system as shown in figure 1. Cold mirrors have many practical applications such as in projectors, copy machines, medical instruments and fibre optical illuminations $[6,7]$.

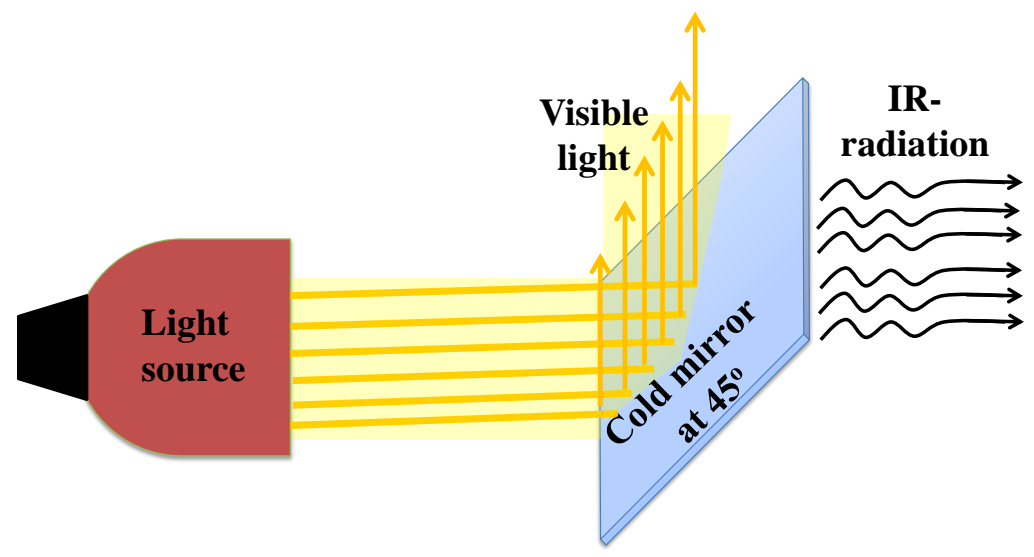

Fig. 1. Schematic of a cold mirror.

\section{Theoretical basis of multilayer structure}

Consider a multilayer dielectric system surrounded by an environment. Light from the source falls on the system at an angle $\alpha_{0}$. For this purpose, wave front can be considered as planar. To calculate the spectral transmittance and reflectance intensity for the p- and s-polarized light, matrix method is used:

$$
T_{s}(\lambda)=\frac{n_{m}}{n_{o}}\left|t_{s}\right|^{2} \quad, R_{s}(\lambda)=\left|r_{s}\right|^{2}
$$




$$
T_{p}(\lambda)=\frac{n_{m}}{n_{o}}\left|t_{p}\right|^{2}, R_{p}(\lambda)=\left|r_{p}\right|^{2}
$$

where $t_{s}, r_{s}$ - amplitude transmission and reflection coefficients of the multilayer interference system for s-polarized light whereas $t_{p}, r_{p}$ - transmission and amplitude reflection coefficients for p-polarized light. Now, we will only consider $s-$ polarization because equations for both $s$ and p polarization are related till equation (7). Amplitude coefficients are determined from the following equations:

$$
\begin{gathered}
t_{s}=\frac{2 n_{o}}{n_{o} m_{11 s}+i n_{o} n_{m} m_{12 s}+i m_{21 s}+n_{m} m_{22 s}}, \\
r_{s}=\frac{n_{o} m_{11 s}+i n_{o} n_{m} m_{12 s}-i m_{21 s}-n_{m} m_{22 s}}{n_{o} m_{11 s}+i n_{o} n_{m} m_{12 s}+i m_{21 s}+n_{m} m_{22 s}},
\end{gathered}
$$

where $\mathrm{n}_{0}, \mathrm{n}_{\mathrm{m}}$ - the effective refractive indices of the substrate and the environment, respectively; $\mathrm{m}_{\mathrm{i}}, \mathrm{j}_{\mathrm{s}}-$ elements of the characteristic matrix $\mathrm{M}_{\mathrm{s}}$ for s-polarized light:

$$
M_{s}=\left(\begin{array}{cc}
m_{11 s} & i m_{12 s} \\
i m_{21 s} & m_{22 s}
\end{array}\right)=M_{1 s} M_{2 s} M_{3 s} \ldots . . M_{q-2 s} M_{q-1 s} M_{q s}
$$

$q$ - Number of layers.

In the expression (5) matrices $\mathrm{M}_{k}(k=\overline{1, q})$ determine the properties of each individual layer of the optical filter. Filter design needs layers with high and low refractive indices. Therefore, the spectral characteristics are described by matrices multiplying:

$$
\begin{gathered}
M_{1}=\left(\begin{array}{cc}
\cos \left(\phi_{1}\right) & \frac{i}{n_{1}} \sin \left(\phi_{1}\right) \\
\operatorname{in}_{1} \sin \left(\phi_{1}\right) & \cos \left(\phi_{1}\right)
\end{array}\right), \quad M_{2}=\left(\begin{array}{cc}
\cos \left(\phi_{2}\right) & \frac{i}{n_{2}} \sin \left(\phi_{2}\right) \\
\operatorname{in}_{2} \sin \left(\phi_{2}\right) & \cos \left(\phi_{2}\right)
\end{array}\right), \\
M_{q-1}=\left(\begin{array}{cc}
\cos \left(\phi_{q-1}\right) & \frac{i}{n_{q-1}} \sin \left(\phi_{q-1}\right) \\
\text { in }_{q-1} \sin \left(\phi_{q-1}\right) & \cos \left(\phi_{q-1}\right)
\end{array}\right), \quad M_{p}=\left(\begin{array}{cc}
\cos \left(\phi_{q}\right) & \frac{i}{n_{q}} \sin \left(\phi_{q}\right) \\
\operatorname{in}_{q} \sin \left(\phi_{q}\right) & \cos \left(\phi_{q}\right)
\end{array}\right),
\end{gathered}
$$

where $\varphi_{\mathrm{k}}$ - phase thickness for s- polarized light, which is calculated by the following equations:

$$
\begin{aligned}
& \phi_{1}=\frac{2 \pi}{\lambda} \mathrm{n}_{1} \mathrm{~h}_{1} \cos \left(\alpha_{1}\right), \quad \phi_{2}=\frac{2 \pi}{\lambda} \mathrm{n}_{2} \mathrm{~h}_{2} \cos \left(\alpha_{2}\right), \\
& \phi_{q-1}=\frac{2 \pi}{\lambda} \mathrm{n}_{q-1} \mathrm{~h}_{q-1} \cos \left(\alpha_{q-1}\right), \quad \phi_{q}=\frac{2 \pi}{\lambda} \mathrm{n}_{q} \mathrm{~h}_{q} \cos \left(\alpha_{q}\right),
\end{aligned}
$$

where $h_{k}$ - the physical thickness of the layers, $n_{m} ; \alpha_{k}-$ the angles of refraction in the layers; $n_{k}-$ effective indexes refractive of the layers which depends on the wavelength. In this case, the angle of refraction in the layers is 45 degrees, relative to the normal.

The main difference in calculations between s- and p- polarized light is specified in (8) and (9) equations.

$$
\begin{aligned}
& \mathrm{n}_{1 s}=\mathrm{n}_{1} \cos \left(\alpha_{1}\right), \quad \mathrm{n}_{2 s}=\mathrm{n}_{2} \cos \left(\alpha_{2}\right), \\
& \mathrm{n}_{1 p}=\mathrm{n}_{1} / \cos \left(\alpha_{1}\right), \mathrm{n}_{2 p}=\mathrm{n}_{2} / \cos \left(\alpha_{2}\right),
\end{aligned}
$$

The angle of refraction in the layers is calculated by the equations (10). 


$$
\alpha_{1}=\arccos \left(\sqrt{1-\frac{n_{o}^{2}}{n_{1}^{2}}} \sin ^{2}\left(\alpha_{o}\right)\right), \alpha_{2}=\arccos \left(\sqrt{1-\frac{n_{o}^{2}}{n_{2}^{2}}} \sin ^{2}\left(\alpha_{o}\right)\right),
$$

Transmission of an unpolarized light is calculated as an average of $\mathrm{T}_{\mathrm{s}}$ and $\mathrm{T}_{\mathrm{p}}$ :

$$
\mathrm{T}=\frac{1}{2}\left(\mathrm{~T}_{s}+\mathrm{T}_{p}\right)
$$

By using these equations, the transmission spectrum of the multilayer $\mathrm{TiO}_{2} / \mathrm{MgF}_{2}$ filter was plotted with the help of Java programing along with the transmission spectrum generated by commercially available open source filter Open filter. Their response is fairly comparable as shown in figure 2.

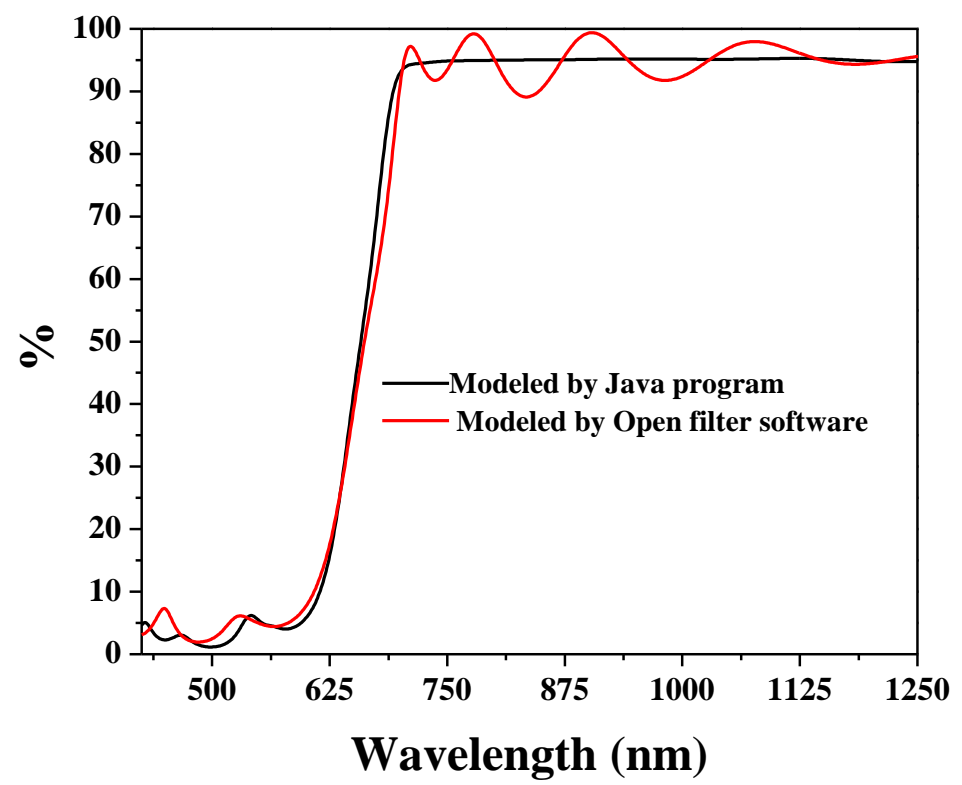

Fig. 2. Transmission spectrum of cold mirror modeled by Java programming and open source software: Open filter.

\section{Filter design}

In the designing of optical filters, the behaviour of the total multilayer system is estimated on the basis of the properties of the individual layers in the stack [8]. Therefore to achieve the optimum performance, it is significant to optically characterize and accurately determine the thickness of the individual layers. In this work, cold mirrors are designed in the wavelength range of 425-1500nm by using open source software Open Filter to selectively pass the wavelengths of interest and rejecting the undesired wavelengths in the visible spectrum. $\mathrm{TiO}_{2}, \mathrm{SiO}_{2}$ and $\mathrm{MgF}_{2}$ materials are carefully selected based on their high and low refractive indices, respectively. $\mathrm{TiO}_{2}$ is a vital dielectric material with a wide band-gap energy and high refractive index that can make it useful in the fabrication of multilayer thin films due to its high optical properties. For instance, its high transmittance and high refractive index in the visible region $(380-760 \mathrm{~nm})$ make it valuable to be employed in the production of the optical filter and window glazing [9, 10]. Layers made of oxides are harder than those made of fluorides, sulphides or semiconductors. Thus, they are ideal to be used on exposed surfaces. Semiconductor materials should be avoided in filters which have to be used over a wide range of temperatures because their optical constants can change considerably. The open filter uses transfer matrix method to analyze the transmission and reflection of light from layers based on thickness and type of materials. Designs are optimized to maximum the transmission required at wavelengths using needle synthesis method (addition of thin layers called needle and analyze transmission till the best results obtained) [11]. The thicknesses of the layers for cold mirror based on $\mathrm{TiO}_{2} / \mathrm{MgF}_{2}$ and $\mathrm{TiO}_{2} / \mathrm{SiO}_{2}$ are shown in table 1 . Both mirrors have 20 layers with almost comparable total thickness. Special attention has been given to keep the thickness of the filters within economic limits.

Assuming the incident angle of un-polarized light equals $45^{\circ}$, these mirrors have reflective properties in the spectral range from $425-610 \mathrm{~nm}$ and $710-1500 \mathrm{~nm}$ up to $95 \%$ and $5 \%$, respectively as shown in figure 3 . For all dielectric stack filters, the transmission depends on the angle of incidence. The central wavelength of the FP filter shifts toward the smaller wavelengths as the angle of incidence is increased. When the incident angle of light decreases from $45^{\circ}$ to $0^{\circ}$ the transmission spectrum shifts from 710 to $750 \mathrm{~nm}$. 


\section{Conclusion}

In this work, we presented the modeling results of cold mirrors based on $\mathrm{TiO}_{2} / \mathrm{MgF}_{2}$ and $\mathrm{TiO}_{2} / \mathrm{SiO}_{2}$ for $45^{\circ}$ of un-polarized incident light by using java programming and commercially available Open source software Open filter. Both mirrors show the reflection of $95 \%$ in the spectral range of $425-610 \mathrm{~nm}$ and $95 \%$ of transmission in the spectral range of 710-1500 $\mathrm{nm}$. The designs are optimized to maximum the transmission required at wavelengths using needle synthesis method. We observed a right shift in a spectrum when the angle of incidence of light was reduced from $45^{\circ}$ to $0^{\circ}$.

\begin{tabular}{cccccc}
\multicolumn{5}{c}{ Table 1. Layer thicknesses of $\mathrm{TiO}_{2} / \mathrm{MgF}_{2}$ and $\mathrm{TiO}_{2} / \mathrm{SiO}_{2}$ based Cold mirrors. } \\
\hline Layer no. & Material & Thickness $(\mathrm{nm})$ & Layer no. & Material & Thickness $(\mathrm{nm})$ \\
\hline 1 & $\mathrm{TiO}_{2}$ & 14 & 1 & $\mathrm{TiO}_{2}$ & 25 \\
2 & $\mathrm{MgF}_{2}$ & 114 & 2 & $\mathrm{SiO}_{2}$ & 121 \\
3 & $\mathrm{TiO}_{2}$ & 45 & 3 & $\mathrm{TiO}_{2}$ & 55 \\
4 & $\mathrm{MgF}_{2}$ & 84 & 4 & $\mathrm{SiO}_{2}$ & 82 \\
5 & $\mathrm{TiO}_{2}$ & 62 & 5 & $\mathrm{TiO}_{2}$ & 55 \\
6 & $\mathrm{MgF}_{2}$ & 71 & 6 & $\mathrm{SiO}_{2}$ & 65 \\
7 & $\mathrm{TiO}_{2}$ & 43 & 7 & $\mathrm{TiO}_{2}$ & 44 \\
8 & $\mathrm{MgF}_{2}$ & 87 & 8 & $\mathrm{SiO}_{2}$ & 99 \\
9 & $\mathrm{TiO}_{2}$ & 44 & 9 & $\mathrm{TiO}_{2}$ & 51 \\
10 & $\mathrm{MgF}_{2}$ & 107 & 10 & $\mathrm{SiO}_{2}$ & 110 \\
11 & $\mathrm{TiO}_{2}$ & 66 & 11 & $\mathrm{TiO}_{2}$ & 71 \\
12 & $\mathrm{MgF}_{2}$ & 90 & 12 & $\mathrm{SiO}_{2}$ & 92 \\
13 & $\mathrm{TiO}_{2}$ & 68 & 13 & $\mathrm{TiO}_{2}$ & 72 \\
14 & $\mathrm{MgF}_{2}$ & 130 & 14 & $\mathrm{SiO}_{2}$ & 123 \\
15 & $\mathrm{TiO}_{2}$ & 48 & 15 & $\mathrm{TiO}_{2}$ & 54 \\
16 & $\mathrm{MgF}_{2}$ & 118 & 16 & $\mathrm{SiO}_{2}$ & 106 \\
17 & $\mathrm{TiO}_{2}$ & 87 & 17 & $\mathrm{TiO}_{2}$ & 93 \\
18 & $\mathrm{MgF}_{2}$ & 54 & 18 & $\mathrm{SiO}_{2}$ & 53 \\
19 & $\mathrm{TiO}_{2}$ & 79 & 19 & $\mathrm{TiO}_{2}$ & 80 \\
20 & $\mathrm{MgF}_{2}$ & 228 & 20 & $\mathrm{SiO}_{2}$ & 218 \\
Total thickness & 1639 & $\mathrm{Total}$ & thickness & 1669 \\
\hline
\end{tabular}

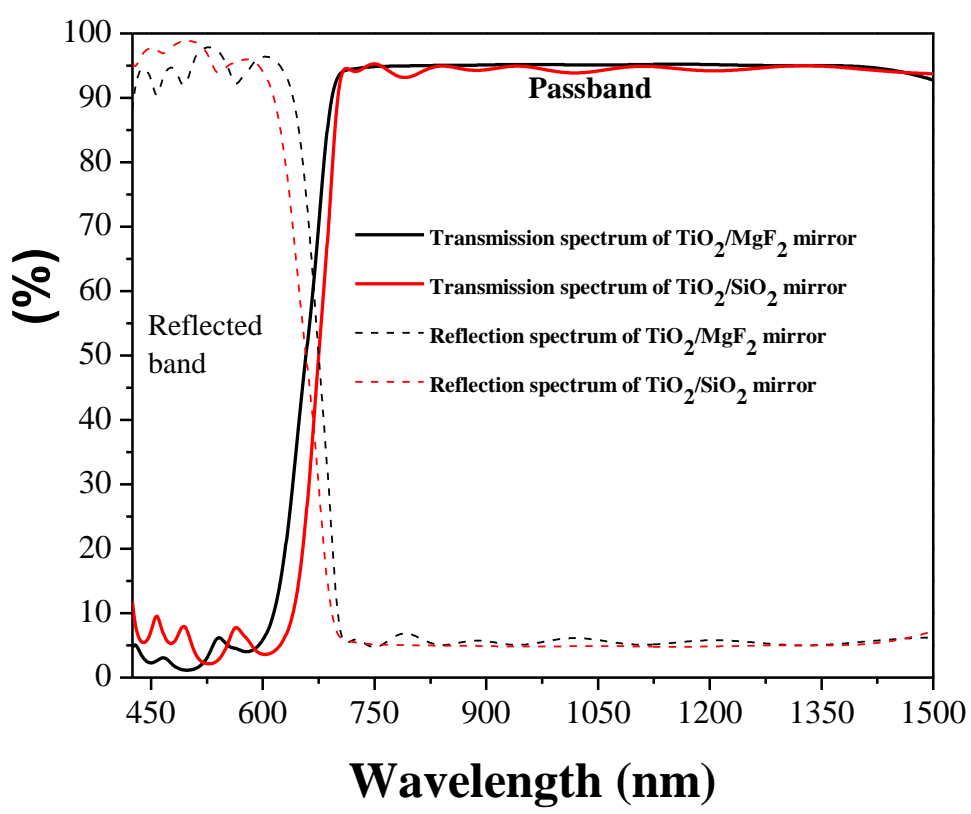

Fig. 3. Transmission and reflection spectrum of the cold mirror in the wavelength range of $425-1500 \mathrm{~nm}$.

\section{Acknowledgements}

This work was supported by the Ministry of Education and Science of the Russian Federation and the Russian Foundation for Basic Research (grant No. 16-29-11698-ofi_m, 16-29-11744-ofi_m).

\section{References}

[1] Macloed HA. Thin film optical filters. McGraw-Hill, 1989

[2] Kazanskiy NL, Serafimovich PG, Popov SB, Khonina SN. Using guided-mode resonance to design nano-optical spectral transmission filters. Computer Optics 2010; 34(2): 162-168.

[3] Kazanskiy NL, Kharitonov SI, Khonina SN, Volotovskiy SG, Strelkov YuS. Simulation of hyperspectrometer on spectral linear variable filters. Computer Optics 2014; 38(2): 256-270.

$3^{\text {rd }}$ International conference "Information Technology and Nanotechnology 2017" 
[4] Kazanskiy NL, Kharitonov SI, Khonina SN, Volotovskiy SG. Simulation of spectral filters used in hyperspectrometer by decomposition on vector Bessel modes, Proc. of SPIE 2015; 9533: 95330L-7pp.

[5] Butt MA, Fomchenkov SA, Ullah A, Habib M, Ali RZ. Modelling of multilayer dielectric filters based on $\mathrm{TiO}_{2} / \mathrm{SiO}_{2}$ and $\mathrm{TiO}_{2} / \mathrm{MgF}_{2}$ for fluorescence microscopy imaging. Computer Optics 2016; 40(5): 674-678. DOI: 10.1109/ICECUBE.2016.7495230.

[6] Baumeister PW. Optical coating technology. SPIE Press book, 2004.

[7] Guenther BD. Modern Optics. Oxford University Press, 2015.

[8] Hinczeweski DS, Hinczeweski M, Tepehen FZ, Tepehen GG. Optical filters from $\mathrm{SiO}_{2}$ and $\mathrm{TiO}_{2}$ multi-layers using sol-gel spin coating method. Solar Energy Materials and Solar Cells 2005; 87(1-4): 181-196.

[9] Hasan MM, Malek ABM, Haseeb ASMA, Masjuki HH. Investigations on $\mathrm{TiO}_{2}$ and Ag based single and multilayer films for window glazings. ARPN Journals of Engineering and Applied Sciences 2010; 5: 22-28.

[10] Butt MA, Fomchenkov SA. Thermal effect on the optical and morphological properties of $\mathrm{TiO}_{2}$ thin films obtained by annealing a Ti metal layer. J. Korean Phys. Soc. 2017; 70(2): 169-172.

[11] Larouche S, Martinu L. Optical filters: Open-source software for the design, optimization, and synthesis of optical filter. Appl. Opt. 2008; 47(13): C219_ C230. 